\title{
Molecular Diagnosis of Plasma Phenylalanine in Neonates with Phenylketonuria Disease Using Biological Sensors Based on Surface-Enhanced Raman Spectroscopy (SERS)
}

\author{
Vahid Eskandari ${ }^{\mathrm{a}}$ and Nafiseh Sharifi ${ }^{\mathrm{b}, *}$ \\ a Nanoscience and Nanotechnology Research Center, University of Kashan, Kashan, Iran \\ ${ }^{b}$ Photonic and Plasma group, Physics Department, University of Kashan, Kashan, Iran \\ Corresponding author Email: sharifi@kashanu.ac.ir
}

\begin{abstract}
Regular paper: Received: Jul. 2, 2019, Revised: Sep. 24, 2019, Accepted: Oct. 27, 2019,
Available Online: June. 30, 2020, DOI: 10.29252/ijop.14.1.31
\end{abstract}

\begin{abstract}
In this study, silver nanoparticles were chemically synthesized and deposited on glass substrates using a reducing agent of sucrose, at $50^{\circ} \mathrm{C}$. Different characterizations including atomic force microscopy (AFM), field emission scanning electron microscopy (FESEM), and Raman spectroscopy were obtained to study silvery substrates. Then, the silvery substrates were used as the SERS substrates to detect vibrational modes of phenylalanine amino acid up to the concentration of $10^{-7} \mathrm{M}$. The importance of phenylalanine amino acid detection is due to the early diagnosis of phenylketonuria in neonates. Therefore, the blood plasma of a healthy neonate and a neonate with phenylketonuria disease were adsorbed on the SERS substrates. They enhance the intensity of molecular vibration peaks of phenylalanine amino acid of two kinds of blood plasmas. The intensities of molecular vibrations of unhealthy plasma are stronger than healthy plasma due to the higher concentrations of phenylalanine amino acid, which is the sign of Phenylketonuria disease.
\end{abstract}

KEYWORDS: SERS Biological Sensors, Silver Nanostructures, Phenylalanine, Blood Plasma, Phenylketonuria.

\section{INTRODUCTION}

Phenylketonuria is an inborn error of metabolism that causes an amino acid called phenylalanine to build up in the body.
Phenylalanine is used by the brain to produce norepinephrine, a chemical that transmits signals between nerve cells and the brain. It keeps you awake and alert, reduces hunger pains, functions as an antidepressant, and helps improve memory [1]. Phenylketonuria is caused by a defect in the gene that helps create the enzyme needed to break down phenylalanine. It can lead to intellectual disability, seizures, behavioral problems, and mental disorders. It may also result in a musty smell and lighter skin, heart problems, a small head, and low birth weight [2]. An important point in relation to phenylketonuria is that if diagnosed timely, the disease can be treated with a regimen, substitute missed enzymes and hormone therapy to prevent catastrophic complications. As a result, the detection of small amounts of phenylalanine and the early diagnosis of phenylketonuria is important. Various techniques such as Highperformance liquid chromatography (HPLC) [3], tandem mass spectrometry (TMS (MS / MS)) [4], gas chromatography-mass spectrometry (GC-MS) [5], and Surface enhanced Raman spectroscopy (SERS) are used for the diagnosis of phenylketonuria. The SERS technique shows high sensitivity and selectivity for detection of different analytes as well as the other techniques are limited by their high cost $[6,7]$. SERS is an emission technique that includes inelastic scattering of 
incident laser resulting in spectral peaks, due to the vibrational modes of the molecule, that are frequency shifted from the incident light. the Raman signal of analyte molecules is enhanced when they adsorb onto the SERS substrate, which are frequently roughened silver/copper/gold surfaces. The advantages of SERS are the high resolution, ease of sample manipulation, in situ analyte identification, speed of analysis, and the advent of commercially available and portable Raman spectrometers. The sensitivity and spectral selectivity of SERS has made it an attractive technique to detect a wide range of chemical species [8]. In order to successfully detect analytes, the SERS substrates must be reproducible and have a roughened surface to give an enhancement [8]. The roughened surface cause the Raman signals enhance due to the light scattering from roughened surface as well as the enormous intensity of the electromagnetic field above the substrate, which is falls off with the distance normal to the surface $[9,10]$. To make SERS substrates, plasmonic particles such as gold and silver particles are typically synthesized using reduction of $\mathrm{HAuCl}_{4}$ or $\mathrm{AgNO}_{3}$ by sodium triacetate, respectively. Then, they are immobilized on silanized substrates. Here, SERS substrates were simply made during the reduction of silver ions without silanization of substrates. In other word, silver ions are reduced on the glass as well as in the solution to have silver coating and silver nanoparticles, respectively. The silvery substrates were utilized as SERS substrates to detect vibrational modes of phenylalanine amino acid. For this purpose, the blood plasma of a healthy neonate and a neonate with phenylketonuria disease were put on the SERS substrates to be adsorbed in order to investigate the effect of these introduced SERS substrates in diagnosis of Phenylketonuria disease.

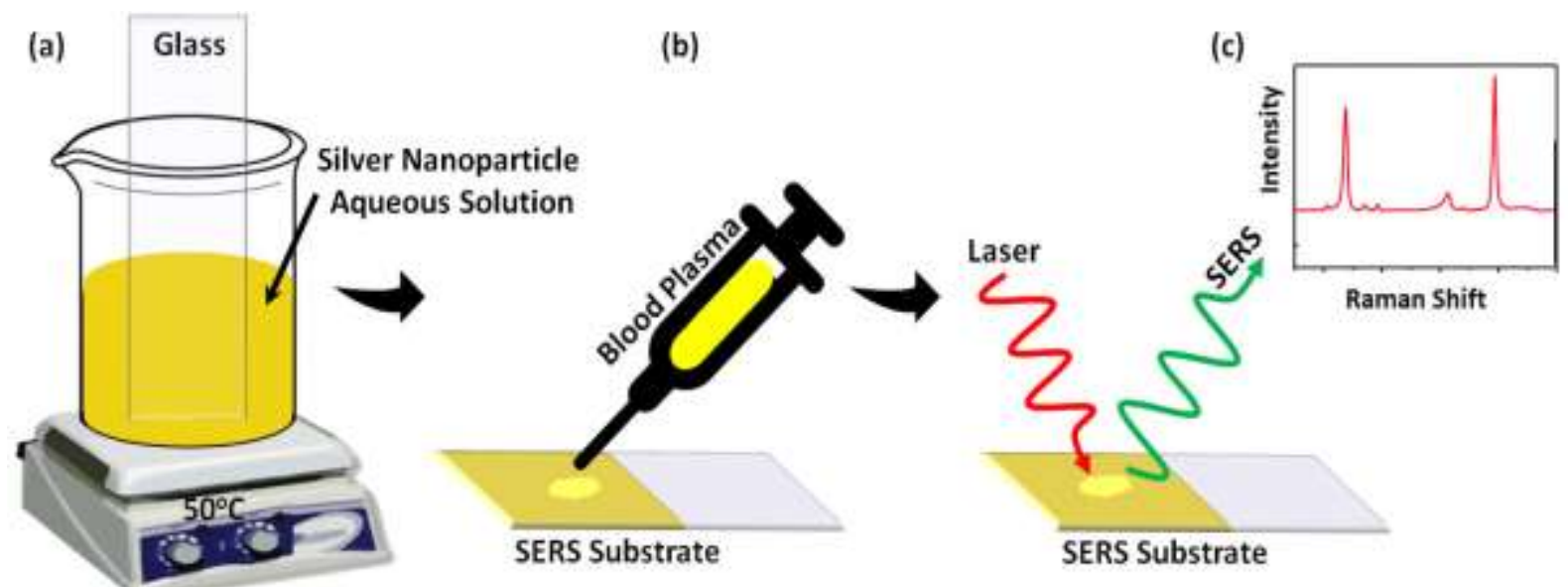

Fig. 1. Schematic of illustration of (a) the fabrication of the SERS substrate, which was fabricated using immersion of a glass in a solution consisting of silver ions and reducing agent, (b) adsorption of blood plasma on the SERS substrate and (c) enhanced Raman signals of blood plasma in vicinity of the SERS substrate.

\section{Experimental Procedure}

\section{A.Fabrication of SERS Substrates and Preparation of Human Blood Plasma Samples}

Glass substrates with the size of $2.5 \mathrm{~cm} \times 1 \mathrm{~cm}$ were cut. Then, silver nanoparticles were chemically coated on the glass substrates. A typical synthesis involves the following steps: ammonium hydroxide (Merck, 25\%) was slowly dropped into $12 \mathrm{~mL}$ of a $0.05 \mathrm{M}$ $\mathrm{AgNO}_{3}$ (Sigma Aldrich) solution. The solution initially turned turbid and then became transparent. The following reactions seem to occur:

$$
\begin{aligned}
& 2 \mathrm{AgNO}_{3}+2 \mathrm{NH}_{4} \mathrm{OH} \rightarrow \\
& \mathrm{Ag}_{2} \mathrm{O}(\mathrm{s}) \downarrow+2 \mathrm{NH}_{4} \mathrm{NO}_{3}+\mathrm{H}_{2} \mathrm{O}
\end{aligned}
$$




$$
\begin{aligned}
& \mathrm{Ag} 2 \mathrm{O}+4 \mathrm{NH} 4++2 \mathrm{OH}-\rightarrow \\
& 2[\mathrm{Ag}(\mathrm{NH} 3) 2]++3 \mathrm{H} 2 \mathrm{O}
\end{aligned}
$$

The solution turned dark brown, after adding some drops of a $0.5 \mathrm{M} \mathrm{KOH}$ (Ghatran Shimi Tajhiz) solution. It was made transparent again by addition of ammonium hydroxide. The substrates were put in the resulting solution. 10 $\mathrm{mL}$ of a solution of $0.07 \mathrm{M}$ sucrose $\left(\mathrm{C}_{12} \mathrm{H}_{22} \mathrm{O}_{11}\right.$, Merck) was then added. Silver complexes were reduced on the surface of the substrates and silver particles were formed $[11,12]$.

$$
\begin{aligned}
& \mathrm{Ag}(\mathrm{NH} 3) 2+(\mathrm{aq})+\mathrm{RCHO}(\mathrm{aq}) \rightarrow \\
& \mathrm{Ag}(\mathrm{s})+\mathrm{RCOOH}(\mathrm{aq})
\end{aligned}
$$

where $\mathrm{R}$ represents $\mathrm{C}_{11} \mathrm{H}_{21} \mathrm{O}_{10}$. The substrates were then removed from the solution and were rinsed with water. Silvery substrates (SERS substrates) were dried at room temperature.

A solution of $10^{-2} \mathrm{M}$ of phenylalanine amino acid in distilled water was prepared. This base solution was diluted to set the concentrations of $10^{-3}, 10^{-4}, 10^{-5}, 10^{-6}$ and $10^{-7} \mathrm{M}$.

Whole blood samples from a healthy neonate and a neonate with phenylketonuria disease were collected in tubes. After a few minutes, when the blood samples coagulate, the blood clots were stirred with a glass rod. Then, they were centrifuged at a speed of $3000 \mathrm{rpm}$ for 7 minutes. Plasmas are the light-yellow liquid on top of the tubes, which were stored at $5-6^{\circ} \mathrm{C}$ before Raman measurements.

\section{B.Sample Preparation for SERS Measurement}

The SERS was checked by depositing a drop of $5 \mu \mathrm{l}$ phenylalanine or $5 \mu \mathrm{l}$ blood plasma aqueous solution on the SERS substrates and observing the Raman response in the range of 0 to $4000 \mathrm{~cm}^{-1}$.

\section{C.Characterization Methods}

Atomic force microscopy (AFM) images were obtained using a Nano System Pars. Postprocessing Image-Plus software (version 2.9) was used to elaborate the AFM image. The surface morphology and composition were investigated using scanning electron microscopy (SEM) (Hitachi, S4160). Raman and SERS spectra of phenylalanine amino acid and blood plasma on SERS substrates were measured using Takram-P50C0R10 equipped with the Nd:YAG laser emitting $532 \mathrm{~nm}$ with $75 \mathrm{~mW}$ incident power.

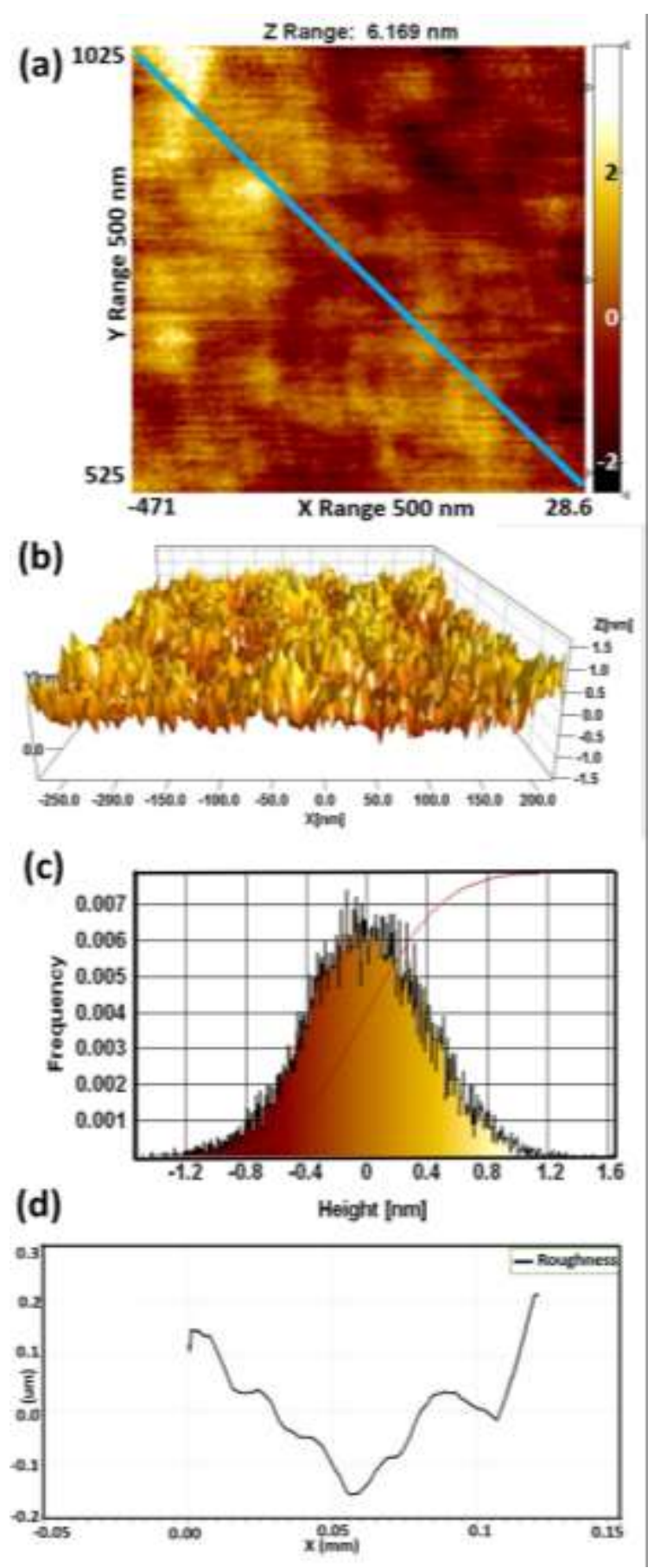

Fig. 2. (a) two- and (b) three-dimensional AFM profiles of the SERS substrate. (c) Height 
distribution function and equivalent Gaussian of and (d) Height profile of the part of the SERS substrate, which has been shown with the blue line in AFM image (a).

\section{RESULTS AND DisCUSSION}

Fig 2. (a) and (b) shows the two and threedimensional surface profiles. The surface morphology of the SERS substrate was observed at an area of $500 \times 500 \mathrm{~nm}^{2}$. The SERS substrate appears average maximum height of the roughness of about $32 \mathrm{~nm}$. Average maximum roughness valley depth as well as average maximum roughness peak height are about 15 and $16 \mathrm{~nm}$, respectively. Its surface roughness could be defined as root mean square roughness, RMS, which was calculated about $11 \mathrm{~nm}$, thus, a marked Raman enhancement for adsorbed molecules is expected by SERS effect, especially employing green laser for Raman excitation [13], normalized histogram and average roughness, respectively. In Fig. 2 (c), height distribution function and equivalent Gaussian of the part of the SERS substrate, which has been marked with the blue line, show that the surface is normal and it is free of bumps and holes in and on the surface. In other words, the surface of the SERS substrates is almost smooth. The positive Skewness of 0.009 are obtained for the SERS substrate, which is attributed to the larger number of peaks on the surface. As we know the negative Skewness is due to a larger number of valleys. Fig. 2 (d) shows height profile for AFM image in Fig. 2 (a). It shows non-intentional alignment for the SERS coting, in which the deepest valley and the highest peak are about 160 and $200 \mathrm{~nm}$, respectively [14].

The FESEM image of phenylalanine amino acid with $10^{-2} \mathrm{M}$ concentration, which was deposited on the SERS substrates are observed in Fig. 3(a). It shows that these substrates have been extremely coated with phenylalanine. The parts of the image which are seen white or brighter are silver coatings because silver is a conductive material and phenylalanine is not.
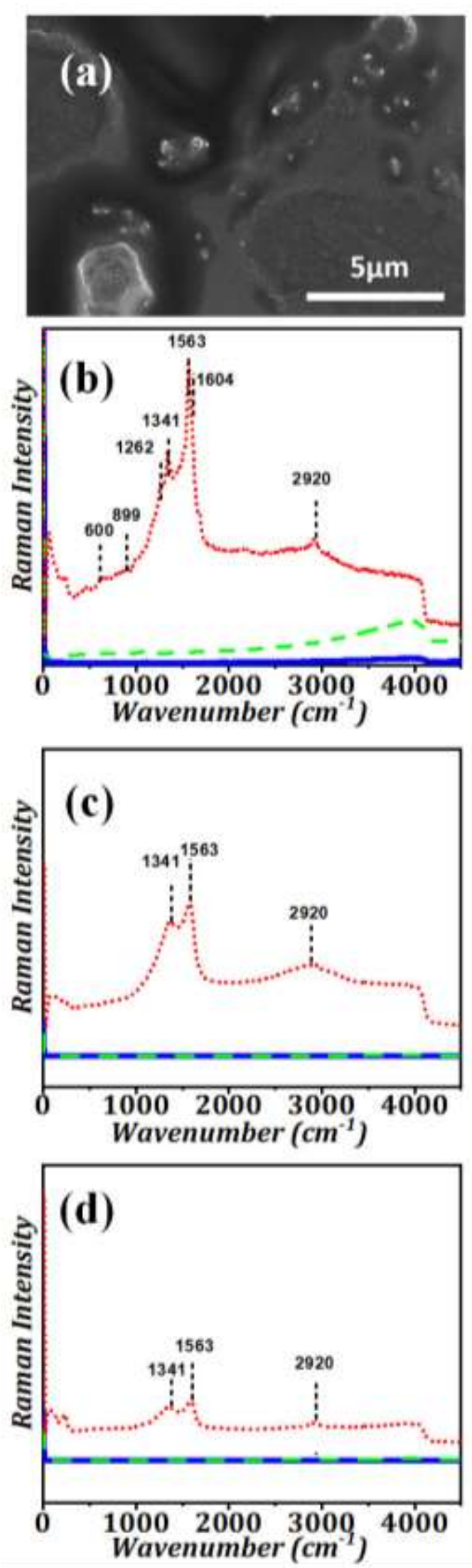

Fig. 3. (a) FESEM image of $10^{-?} \mathrm{M}$ phenylalanine amino acid on the SERS substrate. Raman spectra of (blue-solid curves) the SERS substrate, (greendash curves) phenylalanine on glass substrates; and SERS spectra of (red-dot curves) phenylalanine on the SERS substrates. The concentrations of 
phenylalanine were (b) $10^{-3} \mathrm{M}$, (c) $10^{-5} \mathrm{M}$ and (d) $10^{-7} \mathrm{M}$.

More secondary electrons emitted from silver rather than phenylalanine in FESEM instrument and the more electrons make images white or brighter. The white spots or larger white parts are silver particles which have not completely buried under phenylalanine. The white and large particle, which is seen in the lower left of the image, is about $4 \mathrm{um}$ and it is a coagulation of smaller silver nanoparticles. Larger objects have a high cross-section and more light hits them; thus, more light is scattered [15]. In Fig. 3, Raman spectra of (blue-solid curves) the SERS substrate and (green-dash curves) phenylalanine on glass substrates are seen. The SERS spectra of (red-dot curves) phenylalanine on the SERS substrates are also observed. The concentrations of phenylalanine are $10^{-3} \mathrm{M}, 10^{-5} \mathrm{M}$ and $10^{-7} \mathrm{M}$ in Fig. 3 (b), (c) and (d), respectively. Numbers in Fig. 3 (b), show different molecular vibrations of phenylalanine [1]. phenylalanine has a number of aromatic ring vibrations that their bands appear at 620, 1002, 1033, 1184, 1207, 1585, and $1605 \mathrm{~cm}^{-1}$ are assigned to the in-plane ring deformation $\left(v_{6 b}\right)$, symmetric ring breathing $\left(v_{12}\right)$, in-plane $\mathrm{CH}$ bending $\left(v_{18 \mathrm{a}}\right)$, combination of in-plane $\mathrm{CH}$ bending $\left(v_{9 \mathrm{a}}\right)$ with ring stretching, phenyl-C stretching $\left(v_{7 \mathrm{a}}\right)$, and two in-plane ring stretching vibrations $\left(v_{8 b}\right.$ and $v_{8 \mathrm{a}}$ ), respectively. Bands around 925 and 1155 $\mathrm{cm}^{-1}$ and in the $1300-1500 \mathrm{~cm}^{-1}$ region were assigned, respectively, to the $v\left(\mathrm{C}-\mathrm{COO}_{2}\right)$, $v_{\text {as }}\left(\mathrm{C}_{\alpha} \mathrm{CN}\right)$, and $\delta\left(\mathrm{CH}_{2}\right)$ vibrations [1]. The bands at $600,899,1262,1341,1563,1604 \mathrm{~cm}^{-}$ ${ }^{1}$ in the SERS spectrum occur at nearly the same frequencies of the in plane ring deformation $\left(v_{6 b}\right)$, the $v\left(C-\mathrm{COO}_{2}\right), v_{\mathrm{as}}\left(\mathrm{C}_{\alpha} \mathrm{CN}\right)$, combination of in-plane $\mathrm{CH}$ bending $\left(v_{9 a}\right)$ with ring stretching, two in-plane ring stretching vibrations $\left(v_{8 \mathrm{~b}}\right.$ and $\left.v_{8 \mathrm{a}}\right)$, respectively. This suggests that although the phenyl ring of phenylalanine interacts with the SERS substrate, the interaction has minimal effect on the vibrations [1]. Due to the ring stretching vibration at $1604 \mathrm{~cm}^{-1}$, the amide $\mathrm{I}$ is not clearly seen [1]. Clear changes are seen in intensities of the phenyl ring vibration with general enhancement of the spectra due to adsorption of phenylalanine on the SERS substrates [1]. The band near $2920 \mathrm{~cm}^{-1}$ was assigned to the $\mathrm{CH}$ stretching modes [16].
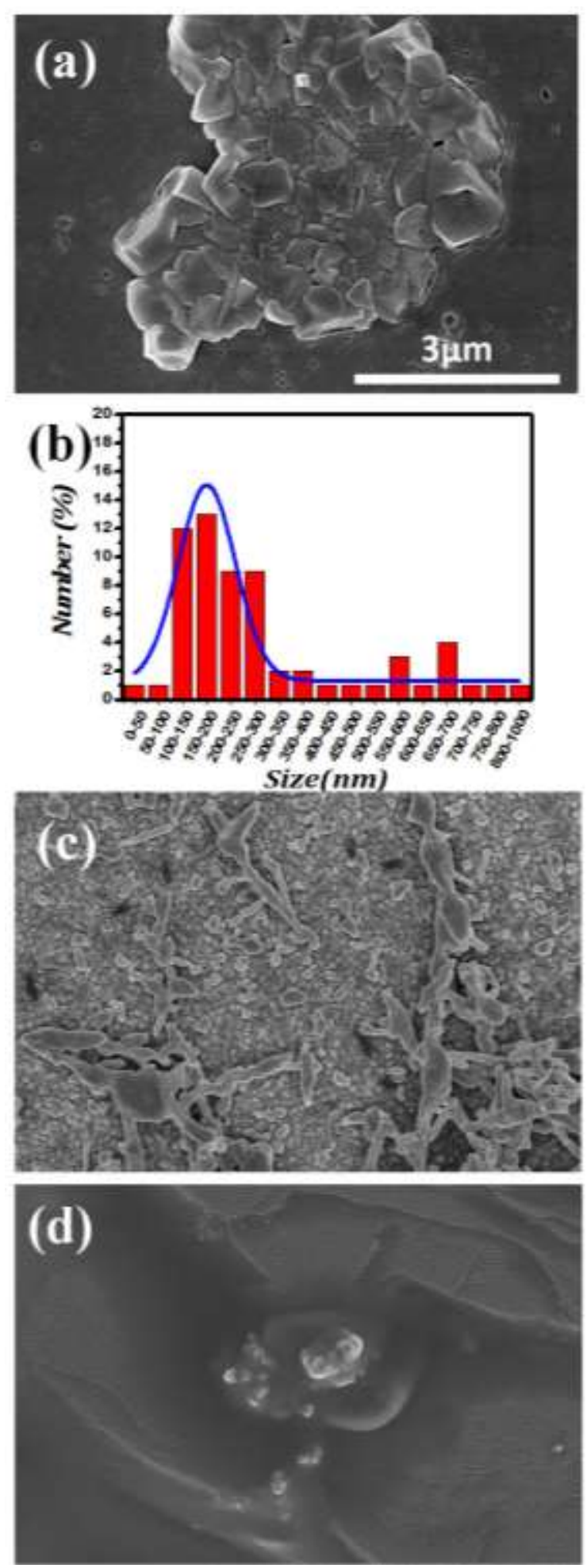

Fig. 4. FESEM images of (a) the SERS substrate. (b) The size distribution of silver larger particles and silver nanoparticles. FESEM images of blood 
plasma on (c) the glass and (d) on the SERS substrate.

Although phenylalanine has no significant signals when it adsorbed on non-SERS substrate (glass), the SERS substrates enhance molecular vibration signals. Since a correlation was established between the wavelength of the incident radiation used for Raman excitation and the size of silver nanoparticles [17]. the enhancement is because of the near field of the silver nanoparticles, which are seen in the coating. In addition, higher cross-section and scattering from larger silver particles (Fig. 3 (a)) enhance the molecular vibrational modes.

As observed in Fig. 3 (c) and (d), the intensities of Raman signals fall with the decrease of phenylalanine concentration. This introduced biosensor could determine phenylalanine up to the concentration of $10^{-7}$ M.

Figure 4 (a) shows the morphology of the SERS substrates. The glass is almost completely covered with silver. Although, the larger particles are seen in middle of the picture, particles smaller than $100 \mathrm{~nm}$ are also observed in the image corners. The most of nanoparticles with the size of smaller than 100 $\mathrm{nm}$, are $20 \mathrm{~nm}$.

The largest observed particle is about $1 \mathrm{um}$. Most of the larger particles are $200 \mathrm{~nm}$. In addition, particles have no specific shapes. The morphologies of blood plasma on the glass and the SERS substrate are seen in Fig. 4 (c) and (d), respectively. They almost completely cover the substrates. Different morphologies of blood plasma are observed since the blood plasma coatings follow the shape of substrates.

Raman spectra of the SERS substrate, blood plasma of a healthy neonate and blood plasma of a neonate with phenylketonuria disease have been shown in Fig. 5 (a). As seen, no specific peaks are observed except a noisy peak at around $2920 \mathrm{~cm}^{-1}$ in spectra of two kinds of blood plasmas deposited on glasses, as previously said this signal was assigned to the $\mathrm{CH}$ stretching modes [16]. In Fig. 5 (b), strong SERS spectra of the blood plasma of a healthy neonate and a neonate with phenylketonuria disease was observed on the SERS substrate by using the $532 \mathrm{~nm}$ laser line, as expected on the basis of AFM and FESEM measurements due to the surface plasmon resonance (near field) of silver nanoparticles and light scattering from the larger silver particles. The $\mathrm{CH}$ stretching modes of phenylalanine at $2920 \mathrm{~cm}^{-1}$ are seen in both spectra. The SERS band at $1580 \mathrm{~cm}^{-1}$ was attributed to the $\mathrm{C}=\mathrm{C}$ bending vibrational mode of phenylalanine [18], and the signal intensity is considerably increased in phenylketonuria disease as seen in Fig 5. (b) since the amount of phenylalanine is higher in blood plasma of neonate with phenylketonuria disease. Bands at around 1004 region were assigned to the symmetric stretch of C-C [18].
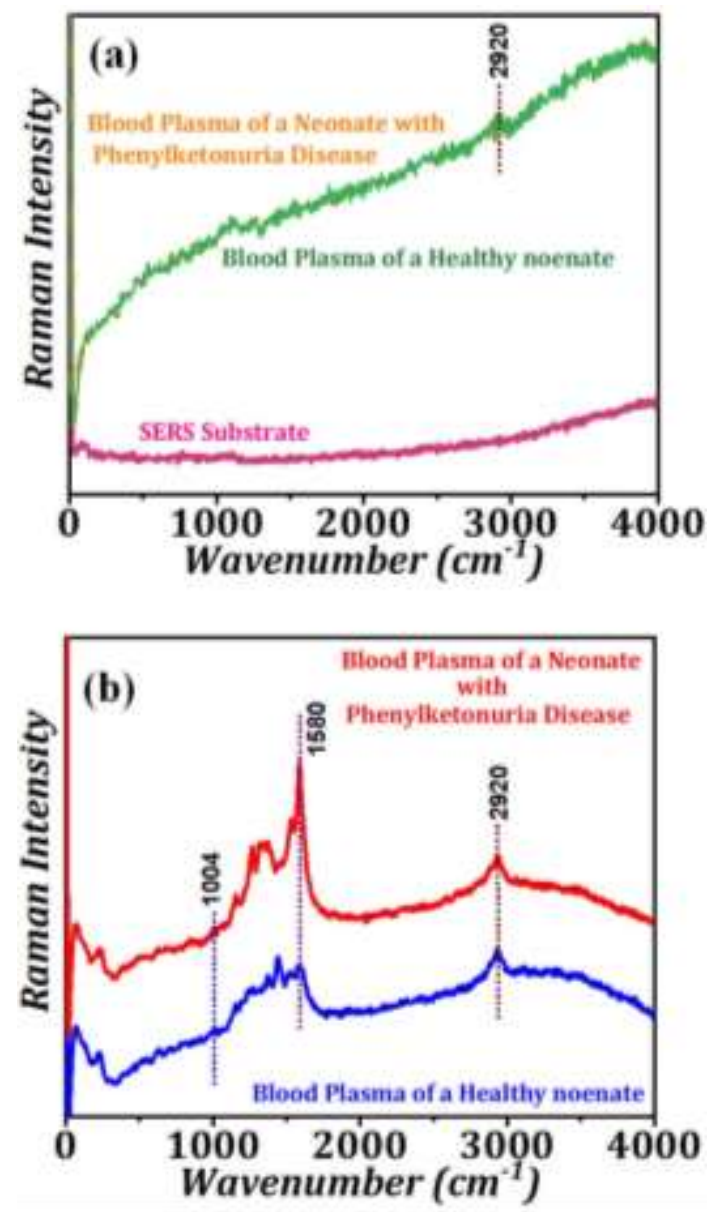

Fig. 5. (a) Raman spectra of (pink) the SERS substrate and two kinds of blood plasmas deposited on glass substrates in which green and orange spectra show Raman of the blood plasma of a healthy neonate and the blood plasma of a neonate 
with phenylketonuria disease, respectively. (b) SERS spectra of (blue) the blood plasma of a healthy neonate and (red) the blood plasma of a neonate with phenylketonuria disease (two kinds of blood plasmas deposited on the SERS substrates).

\section{Conclusion}

In this study, the SERS substrates were made using a chemical method, which is free of surface silanization. Consequently, phenylalanine amino acid as well as the blood plasma of a healthy neonate and the blood plasma of a neonate with phenylketonuria disease was deposited and dried on the SERS substrate. The vibrational modes of phenylalanine in three kinds of samples were detected by Raman spectroscopy. Increase in intensity of vibrational modes of phenylalanine are observed when the SERS substrates are used in Raman spectroscopy. In conclusion, the introduced SERS substrate exhibit a giant Raman amplification of the adsorbed molecules by SERS effect, ensuring important perspective applications in biomedicine.

\section{REFERENCES}

[1] E. Podstawka, Y. Ozaki, and L.M. Proniewicz, "Part I: Surface-Enhanced Raman Spectroscopy Investigation of Amino Acids and Their Homodipeptides Adsorbed on Colloidal Silver," Appl. Spectrosc. Vol. 58, pp. 570-580, 2004.

[2] N. Blau, "Review: Genetics of Phenylketonuria: Then and Now," Human Mutation, Vol. 37, pp. 508-515, 2016.

[3] M.L. Cheng, B.C. Tsai, and J. Yang, "Silver nanoparticle-treated filter paper as a highly sensitive surface-enhanced Raman scattering (SERS) substrate for detection of tyrosine in aqueous solution," Analytica. Chimica. Acta, Vol. 708, pp. 89-96, 2011.

[4] S. Banta-wright and R. Steiner, "Tandem Mass Spectrometry in Newborn Screening Tandem Mass Spectrometry in Newborn Screening," A. Primer. Neonatal. Nurses, Vol. 18, pp. 41-60, 2004.

[5] H.F. Escobar-Morreale, S. Samino, M. Insenser, M. Vinaixa, M. Luque-Ramı'rez,
M.A. Lasuncion, and X. Correig, "Metabolic Heterogeneity in Polycystic Ovary Syndrome Is Determined by Obesity: Plasma Metabolomic Approach Using GC-MS," Clinic. Chem. Vol. 58, pp. 999-1009, 2012.

[6] V.L. Brumm, D. Bilder, and S.E. Waisbren, "Psychiatric symptoms and disorders in phenylketonuria," Mol. Genet. Metab. Vol. 99, pp. 559-563, 2010.

[7] M. Javanmard and R. W. Davis, "SurfaceEnhanced Raman Scattering (SERS) for Detection of Phenylketonuria for Newborn Screening," Soc. Photo-Optical Instrument. Eng. (SPIE), Vol. 8954, pp. 89540R-1 (1-7), 2014.

[8] G. McNay, D. Eustace, W.E. Smith, K. Faulds, and D. Graham, "Surface-Enhanced Raman Scattering (SERS) and SurfaceEnhanced Resonance Raman Scattering (SERRS): A Review of Applications," Appl. Spectrosc, Vol. 65, pp. 825-837, 2011.

[9] G.J. Kovacs, R.O. Loutfy, P.S. Vincett, C. Jennings, and R. Aroca, "Distance Dependence of SERS Enhancement Factor from Langmuir-Blodgett Monolayers on Metal Island Films: Evidence for the Electromagnetic Mechanism," Langmuir, Vol. 2, pp. 689-694, 1986.

[10]P. A. Mosier-Boss, "Review of SERS Substrates for Chemical Sensing," Nano materials for SERS Applications, Vol. 7, pp. 142 (1-30), 2014.

[11] N. Sharifi and N. Taghavinia, "Silver nanoislands on glass fibers using heat segregation method," Mater. Chem. Phys, Vol. 113, pp. 63-66, 2009.

[12] Y. Yin, Z.Y. Li, Z. Zhong, B. Gates, Y. Xia, and S. Venkateswaran, "Synthesis and characterization of stable aqueous dispersions of silver nanoparticles through the Tollens process," J. Mater. Chem. Vol. 12, pp. 522-527, 2002.

[13]E. Giorgetti, S. Cicchi, M. MunizMiranda, G. Margheri, T. D. Rosso, A. Giusti, A. Rindi, G. Ghini, S. Sottini, A. Marcellif, and P. Foggig, "Förster resonance energy transfer (FRET) with a donoracceptor system adsorbed on silver or gold nanoisland films," Phys. Chem. Vol. 11, pp. 9798-9803, 2009. 
[14] V. Nalladega (Ed.) Scanning Probe Microscopy-Physical Property Characterization at Nanoscale, Chap. 3, E.M. Therézio, M.L. Vega, R.M. Faria, and A. Marletta, Statistical Analysis in Homopolymeric Surfaces, InTech, 2012.

[15]C.F. Bohren and D.R. Huffman, Absorption and Scattering of Light by Small Particles, Wiley New York, 1983.

[16] S.Y. Lin, M. J. Li, and W.T. Cheng, "FT-IR and Raman vibrational micro spectroscopies used for spectral bio diagnosis of human tissues," Spectroscopy, Vol. 21, pp. 1-30, 2007.

[17] S. R. Emory, W.E. Haskins, and Sh. Nie, "Direct Observation of Size-Dependent Optical Enhancement in Single Metal Nanoparticles," J. Am. Chem. Soc. Vol. 120, pp. 8009-8010, 1998.

[18] Sh. Feng, R. Chen, J. Lin, J. Pan, G. Chen, Y. Li, M. Cheng, Z. Huang, J. Chen, and H. Zeng, "Nasopharyngeal cancer detection based on blood plasma surface-enhanced Raman spectroscopy and multivariate analysis," Biosensors and Bioelectronics, Vol. 25, pp. 2414-2419, 2010.

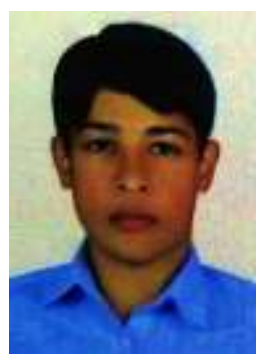

Vahid Eskandari received his B.Sc. degree in nuclear physics from Urmia University in Iran and M.Sc. degree in nanotechnology (nanophysics) from University of Kashan, respectively in 2016 and 2018. He received his M.Sc. degree under the supervision of Dr. Nafiseh Sharifi, assistant professor at University of Kashan. His research focuses on the surface enhanced Raman spectroscopy for biological diagnosis.

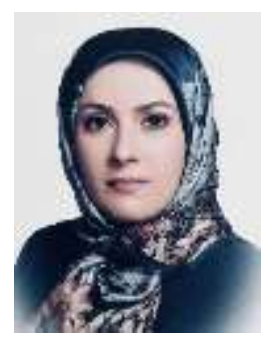

Nafiseh Sharifi received her B.Sc. and M.Sc. degrees in physics from Alzahra University and Sharif University of Technology (SUT), Tehran, Iran, respectively. She obtained her Ph.D. in nanotechnology from Sharif University of Technology in 2012. She was a postdoctoral researcher at Sharif University of Technology until 2013 when she became an assistant professor at University of Kashan. Her research interests include plasmonics, surface enhanced Raman spectroscopy, optoelectronic devices, dye-sensitized solar cells, provskite solar cells and electrochemical sensors. 